\title{
Probing the wave functions of correlated states in magic angle graphene
}

\author{
Zhiming Zhang $\odot,{ }^{1}$ Rachel Myers, ${ }^{1}$ Kenji Watanabe $\odot,{ }^{2}$ Takashi Taniguchi, ${ }^{2}$ and Brian J. LeRoy $\oplus^{1, *}$ \\ ${ }^{1}$ Physics Department, University of Arizona, 1118 East 4th Street, Tucson, Arizona 85721, USA \\ ${ }^{2}$ National Institute for Materials Science, Namiki 1-1, Tsukuba, Ibaraki 305-0044, Japan
}

(Received 19 March 2020; revised 16 June 2020; accepted 8 July 2020; published 3 August 2020)

\begin{abstract}
Using scanning probe microscopy and spectroscopy, we explore the spatial symmetry of the electronic wave functions of twisted bilayer graphene at the "magic angle" of $1.1^{\circ}$. This small twist angle leads to a long wavelength moiré unit cell on the order of $13 \mathrm{~nm}$ and the appearance of two flat bands. As the twist angle is decreased, correlation effects increase until they are maximized at the magic angle. At this angle, the wave functions at the charge neutrality point show reduced symmetry due to the emergence of a charge ordered state. As the system is doped, the symmetry of the wave functions changes at each commensurate filling of the moiré unit cell pointing to the correlated nature of the spin and valley degeneracy broken states.
\end{abstract}

DOI: 10.1103/PhysRevResearch.2.033181

\section{INTRODUCTION}

When two layers of graphene are twisted away from each other at the "magic angle," around $1.1^{\circ}$, the long wavelength moiré pattern leads to the folding of the band structure into a mini-Brillouin zone and the formation of ultraflat bands [1-7]. As the twist angle approaches the magic angle, the bands become flatter and the ratio between the Coulomb interaction and their bandwidth increases, leading to increased correlation effects [8-21] and broken symmetry states [22-24]. Superconductivity and correlated insulating states [25-29] have been discovered in magic angle twisted bilayer graphene (MATBG) when the flat bands are tuned to commensurate filling factors $v=0, \pm 1, \pm 2, \pm 3$ electrons per moiré unit cell. For $v=$ $\pm 1( \pm 3)$, there is only one electron (hole) in one of the flat bands; hence both the spin and valley degeneracy must be broken while for even filling factors one of these degeneracies must be restored. Due to the correlated nature of these states, small changes in the filling factors can lead to dramatic changes in the wave functions. Transport studies [16,27-29] have revealed that the phenomenology of the commensurate states at odd filling levels are different from those at even filling levels. Theory calculations [16,22,30,31] suggest that odd and even filling levels will lead to different symmetry breaking phases.

Since there is still considerable theoretical uncertainty in the exact nature of the wave functions at each of the commensurate fillings, direct imaging of the wave functions can provide experimental insights for explaining the coherent states that have been observed in MATBG. For example, revealing the exact spatial distribution of flat band wave functions at

\footnotetext{
*leroy@ arizona.edu

Published by the American Physical Society under the terms of the Creative Commons Attribution 4.0 International license. Further distribution of this work must maintain attribution to the author(s) and the published article's title, journal citation, and DOI.
}

different commensurate fillings can provide information such as anisotropy and localization properties. Our scanning tunneling microscopy and spectroscopy (STM and STS) study focuses on the difference in the wave functions between even and odd filling factors, which has not been explored by previous STM studies on MATBG [23,24,32-34]. We find that for the partially filled bands, the wave functions are localized on different parts of the moiré unit cell. We further explore the properties of wave functions by quantitatively extracting the anisotropy, localization, and radial distribution for various energy and filling levels, providing a comprehensive local characterization of flat bands in MATBG.

\section{EXPERIMENT}

Figure 1(a) shows a schematic of the experimental setup. All measurements were performed in ultrahigh vacuum at a temperature of $4.6 \mathrm{~K}$. Samples were fabricated by a dry transfer technique with controlled rotational alignment between the two layers of graphene [35]. A bias voltage $\left(V_{b}\right)$ applied between the tip and the sample is used for probing different energy levels, while the gate voltage $\left(V_{g}\right)$ applied between the Si substrate and the sample is used for tuning the doping level of the MATBG. Figure 1(b) shows an STM topography image of a $1.10^{\circ}$ MATBG moire pattern; the twist angle is determined by measuring the moiré wavelengths in three different directions (L1, L2, L3) and using a uniaxial heterostrain model [24]. The bright spots in the topography image correspond to the $A A$ sites of the moiré superlattice; other high-symmetry sites are labeled as $A B, B A$. The atomic arrangements of these high-symmetry points are illustrated in the schematic for a $5^{\circ}$ moiré pattern in Fig. 1(c). Figure 1(d) shows the STS on the $A A$ and $A B$ sites when the flat bands are fully occupied. The two sharp peaks between -0.05 and $0 \mathrm{~V}$ in the spectroscopy of the $A A$ site correspond to the strong local density of states (LDOS) of the flat bands, while the LDOS in this energy range is much weaker on the $A B$ site, indicating that the flat bands are localized on the $A A$ site $[3,5]$. 


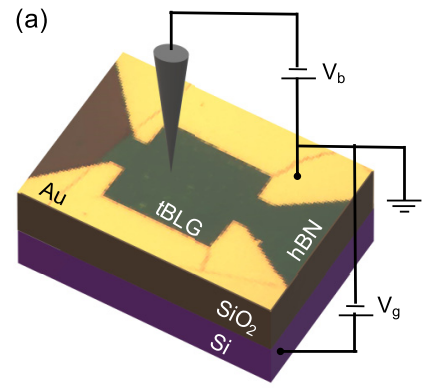

(b)

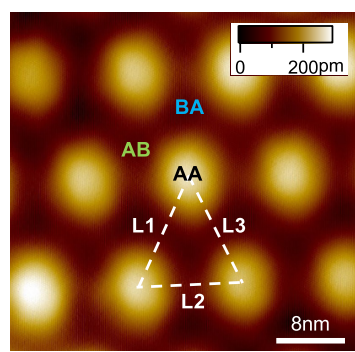

(c)

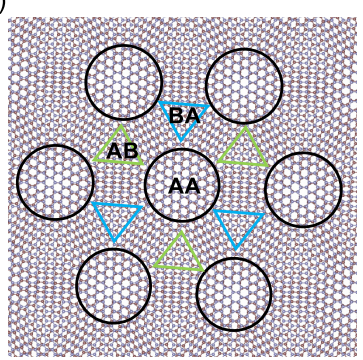

(d)

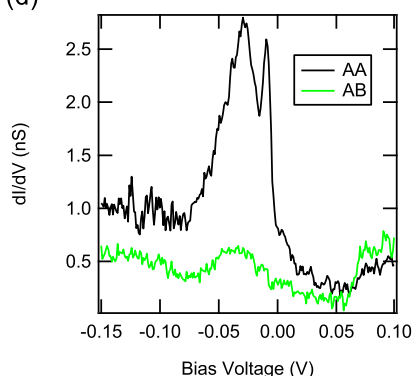

FIG. 1. (a) Schematic of the experimental setup showing the STM tip and an optical microscope image of the measured MATBG sample. (b) Atomic resolution STM topography of the $1.10^{\circ}$ moiré superlattice. (c) Schematic of different atomic stacking arrangements arising from the twist of the two layers of graphene. (d) $d I / d V$ spectroscopy of the MATBG on $A A$ and $A B$ sites of $1.10^{\circ}$ moiré when the flat bands are fully filled, where $V_{g}=8 \mathrm{~V}$.

\section{TUNNELING SPECTROSCOPY}

To study the electronic properties of the flat bands at different doping levels, we performed STS measurements on

the $A A$ site as a function of both $V_{b}$ and $V_{g}$, as shown in Fig. 2(a) for a $1.07^{\circ}$ twist angle moiré. For each $V_{g}$, the tip height was stabilized at a bias voltage of $0.1 \mathrm{~V}$ and tunnel current of $50 \mathrm{pA}$. Then the feedback circuit was switched off, a small ac voltage $(1 \mathrm{mV})$ was applied to the bias voltage, and the differential conductance $d I / d V$ was measured as a function of bias voltage using lock-in detection. The tip gating effect [33] was corrected from the data; see Sec. 1 of the Appendix for details. When the two flat bands are fully filled $\left(V_{g}>14 \mathrm{~V}\right)$ or fully depleted $\left(V_{g}<-50 \mathrm{~V}\right)$, they are close together with a peak to peak separation of $\sim 14 \mathrm{mV}$ and their energy shifts linearly with respect to $V_{g}$ with a slope of $\sim 3 \mathrm{mV}$ per volt in $V_{g}$. In contrast, in the region where the flat bands are overlapping with the Fermi level $\left(-50 \mathrm{~V}<V_{g}<14 \mathrm{~V}\right)$, the energy of the flat bands changes much slower with respect to $V_{g}$. On top of this, various unique features of the flat bands emerge:

(1) The total bandwidth of the flat bands, defined as the full width at the half maximum on either side of the flat bands, indicated in Figs. 2(b) and 2(c), is broadened in this region, shown by the black curve in Fig. 2(f). The overall broadening indicates stronger electron-electron interactions and the breakdown of a single particle picture [30,32]. Such broadening is not present in the spectroscopy of non-magicangle devices, as shown in Sec. 2 of the Appendix.

(2) The separation between the two flat bands is enhanced near the charge neutrality point (CNP, defined as where the conduction band and valence band are equidistant from the Fermi level and have the same peak LDOS) around $V_{g}=$ $-26 \mathrm{~V}$, which is a result of stronger interlayer exchange interactions $[30,32,33]$. To illustrate that this effect is strongest near the magic angle of $1.1^{\circ}$, we measured the total bandwidth at $\mathrm{CNP}\left(W_{\mathrm{CNP}}\right)$ and when both bands are fully depleted $\left(W_{\mathrm{FD}}\right)$
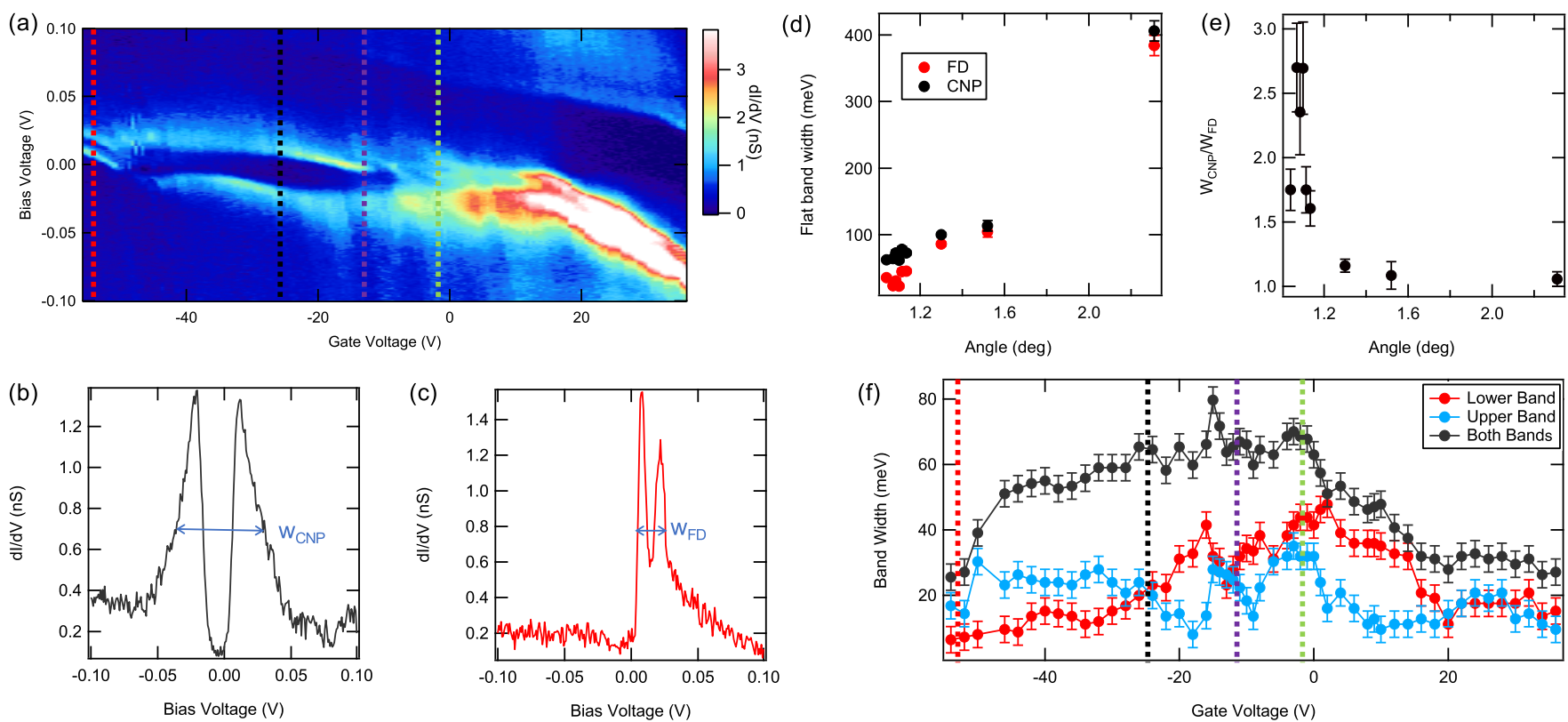

FIG. 2. (a) Gate-dependent STS spectroscopy on the $A A$ site of the $1.07^{\circ}$ moire superlattice. Dashed lines indicate the position of commensurate fillings: $v=-4$ (red), $v=0$ (black), $v=1$ (purple), $v=2$ (green). (b) $d I / d V$ curve at CNP, $V_{g}=-26 \mathrm{~V}$. (c) $d I / d V$ curve at FD, $V_{g}=-54 \mathrm{~V}$. (d) Flat band width at two different fillings as a function of twist angle. (e) The ratio of flat band width at two different fillings as a function of twist angle. (f) The flat band width extracted from (a) as a function of $V_{g}$. 

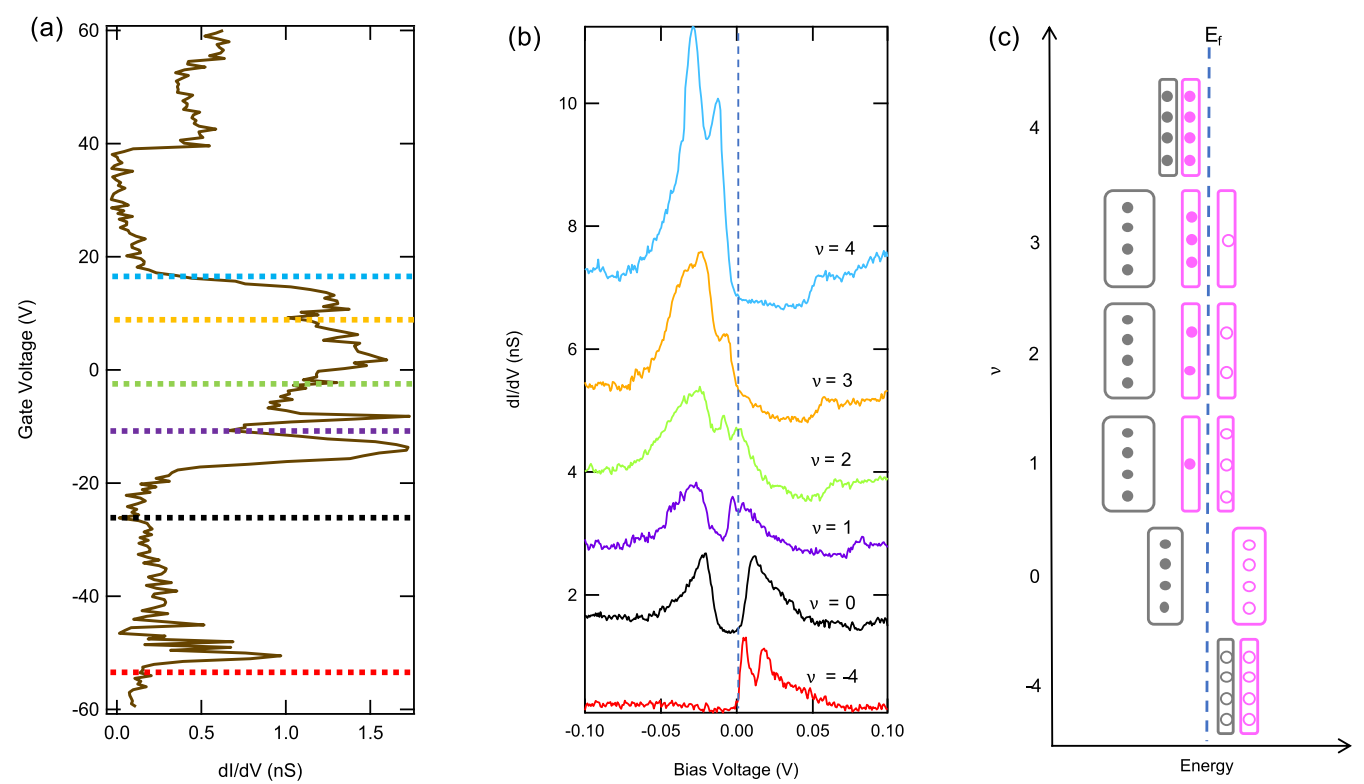

FIG. 3. (a) Gate-dependent LDOS at the Fermi level on the $1.07^{\circ}$ moiré superlattice, colored dashed lines correspond to different commensurate fillings. (b) $d I / d V$ curves at different commensurate fillings, vertical dashed line marks the Fermi level. (c) Illustration of the evolution of the flat bands at different filling levels. Pink and gray correspond to the upper band and lower band, respectively, while solid dots are electrons and circles are holes.

from $d I / d V$ measurements of different twist angle devices. The total bandwidth as a function of angle is plotted in Fig. 2(d); while the bandwidth decreases with the twist angle, the difference between $W_{\mathrm{CNP}}$ and $W_{\mathrm{FD}}$ increases at small angle. As shown in Fig. 2(e), the ratio between $W_{\mathrm{CNP}}$ and $W_{\mathrm{FD}}$ shows a peak around $1.07^{\circ}$, confirming that the exchange interaction is indeed strongest around the magic angle. Other angledependent quantities such as separation between the two flat bands, individual bandwidths, and the separation between the flat bands and remote bands are presented in Sec. 3 of the Appendix.

(3) Near the CNP, the width of the upper conduction band is wider than the lower valence band in the $p$-doped region $\left(V_{g}<-26 \mathrm{~V}\right)$ and vice versa in the $n$-doped region $\left(V_{g}>-26 \mathrm{~V}\right)$; see the red and blue curves in Fig. 2(f). The spectral weight redistribution between the two flat bands as a function of doping is consistent with previous experiments suggesting a correlated charge ordered phase [23]; Mottinsulating states as well as superconductivity near CNP have also been observed in transport measurements [28].

(4) In the range of $-15 \mathrm{~V}<V_{g}<13 \mathrm{~V}$ when the lower band is fully filled and the upper band is partially filled, both bands show various distortions. Dips appear in the LDOS in the upper band at certain doping levels and the lower band is broadened when the upper band is partially filled. To better identify the location of the dips and compare our STS results with transport experiments [25-29], we plot the LDOS at the Fermi level as a function of $V_{g}$ in Fig. 3(a), which is a horizontal line cut from Fig. 2(a) at zero bias voltage. Consistent with transport measurements [25-29], strong insulating states appear when the bands are fully filled $\left(V_{g}>14 \mathrm{~V}\right)$, fully depleted $\left(V_{g}<-50 \mathrm{~V}\right)$, and around the CNP $\left(-46 \mathrm{~V}<V_{g}<-20 \mathrm{~V}\right)$. Additional dips in the LDOS show up around $V_{g}=-12,-1$, and $9 \mathrm{~V}$, similar to the insulating states with filling factors of $v=$ 1,2 , and 3 observed in transport measurements [25-29]. From the individual $d I / d V$ curves at these gate voltages in Fig. 3(b), a soft gaplike structure appeared at the Fermi level for $V_{g}=-12 \mathrm{~V}(v=1)$ and $V_{g}=-1 \mathrm{~V}(v=2)$ but not for $V_{g}=9 \mathrm{~V}(v=3)$.

Figure 3(c) illustrates the evolution of the two flat bands as a function of filling levels; pink and gray rectangles correspond to the upper and lower bands, respectively; solid and empty dots correspond to electrons and holes that are occupying the flat bands. At $v=0$, both bands are broadened, and their separation is enhanced compared to $v= \pm 4$. When the upper band is partially filled $(v=1,2,3)$, the upper band is split into two bands on either side of the Fermi level; meanwhile the lower band is further broadened.

The asymmetry of the $d I / d V$ as a function of $V_{g}$ as well as the missing gap at $v=3$ is because of tip-induced band bending [32]. With different tip conditions, we observed symmetric gate-dependent spectroscopy with no tip-induced bending as well as asymmetric gate-dependent spectroscopy with tip-induced bending in a different direction, as shown in Sec. 4 of the Appendix. The $d I / d V$ signal in Fig. 2(a) is much stronger when the flat bands are fully filled; this is because we are setting the tip at a positive voltage $(0.1 \mathrm{~V})$ when starting to perform the spectroscopy. When the bands are fully filled, there is almost no density of states present from the Fermi level up to the positive tip voltage; thus the tip height will be stabilized closer to the sample in order to reach the set current, which in turn increases the $d I / d V$ signal. By setting the starting voltage at a negative voltage, the $d I / d V$ signal is stronger when the flat bands are fully depleted; see Sec. 4 of the Appendix. 

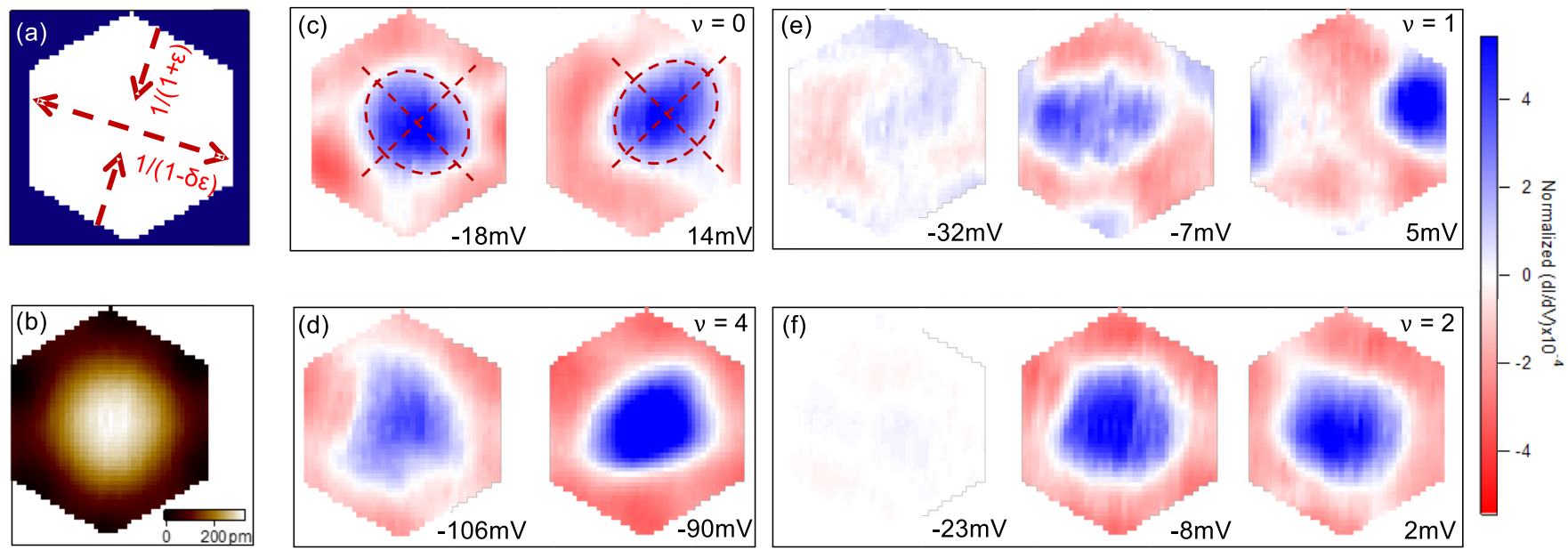

FIG. 4. (a) Uniaxial strain directions relative to the unit cell. (b) Topography of the unit cell. (c) LDOS maps at CNP ( $v=0$ ), dashed ovals highlight the shape of the wave functions, and dashed lines show the symmetry axes. (d) LDOS maps when the upper band is fully filled $(v=4)$. (e) LDOS maps when the upper band has one electron $(v=1)$. (f) LDOS maps when the upper flat band is half filled $(v=2)$.

\section{DENSITY OF STATES MAPS}

Although gap opening and insulating behavior has been observed at all commensurate fillings: $v=0(\mathrm{CNP}), \pm 1$, \pm 2 , and \pm 3 . The intrinsic gap driving mechanism for these states can be very different $[13,16,27,30,31]$. Parallel fielddependent transport measurements [27] have found that the $v= \pm 1$ and $v= \pm 3$ states are spin polarized while the $v=$ \pm 2 states are not. A ferromagnetic state [29] and intrinsic quantized anomalous Hall effect [20] have been found in the $v=3$ state; Chern insulating states have been discovered for the $v= \pm 1$ states [28]. To highlight the difference between the different commensurate filling states, which have not been addressed in the previous STM studies on MATBG [23,24,32-34], we measure the spatial profile of the wave functions at various energy and doping levels by mapping the LDOS with different $V_{b}$ and $V_{g}$.

The LDOS maps at the energies of the flat bands for different commensurate fillings are shown in Fig. 4; each one of them is averaged over several unit cells and plotted in the Wigner-Seitz cell of the moiré lattice centered at the $A A$ site. The $d I / d V$ value is normalized so that the summation of $d I / d V$ over the unit cell is 1 ; then the average value is subtracted. Thus the average value will always appear as white in the color plot, independent of the setting of the color scale. In order to compare these LDOS maps between different fillings, all images are plotted under the same color scale. The strain directions that are extracted from the uniaxial heterostrain model [24] are plotted in Fig. 4(a) with respect to the unit cell, where the strain percentage was found to be $\varepsilon=0.17 \%$ using the Poisson ratio $\delta=0.16$ for graphene. Figure 4(b) shows the unit cell topography of the same area, which has almost full $C_{6}$ symmetry, only slightly altered by the strain. In contrast, the LDOS maps at the CNP, Fig. 4(c), show reduced symmetry with the symmetry axes perpendicular to each other between the lower band $\left(V_{b}=-18 \mathrm{mV}\right)$ and the upper band $\left(V_{b}=14 \mathrm{mV}\right)$, which is a result of a charge ordered state [23]. When both bands are fully filled, Fig. 4(d), similar broken symmetry still exists but the shape of the wave functions for the lower $\left(V_{b}=-106 \mathrm{mV}\right)$ and upper $\left(V_{b}=-90 \mathrm{mV}\right)$ bands are not as perpendicular to each other as they are in Fig. 4(b), indicating the absence of a charge ordered state [23]. LDOS maps at other energies and doping levels, as well as a qualitative study of the anisotropy and localization; are present in Sec. 5 of the Appendix. Interestingly, the nature of the wave functions changes dramatically when the bands are partially filled, as shown in Figs. 4(e) and 4(f) for $v=1$ and $v=2$. The wave functions of the fully filled lower band $\left(V_{b}=-32 \mathrm{mV}\right.$ for $v=1, V_{b}=-23 \mathrm{mV}$ for $\left.v=2\right)$ are much less localized compared to Figs. 4(b) and 4(e). The width of the bands in our spectroscopy measurements is related to their flatness in momentum space. The larger the range of momenta for a given state, the more localized it will be in real space. Thus, the delocalization when the upper band is partially filled is related to the broadening of the lower band as shown previously in Fig. 2. The delocalization of the wave function further supports that the broadening of the flat bands is intrinsic, and is not from tip-induced effects.

For the partially filled upper band, the wave functions are no longer localized at $A A$ sites when $v=1$; the occupied state $\left(V_{b}=-7 \mathrm{mV}\right)$ and the unoccupied state $\left(V_{b}=5 \mathrm{mV}\right)$ are localized on opposite locations somewhere between $A A$ (center of the unit cell) and the boundary of the unit cell. On the other hand, the wave functions of the upper band for $v=2$ are still localized on the $A A$ sites. Compared to Fig. 4(b), these wave functions do not have rotational symmetry and further they are not perpendicular to each other, which provides further evidence that the perpendicularity of wave functions at the CNP originates from a charge ordered state [23], since both the occupied and unoccupied wave functions here at $v=2$ are from the upper band. The stark difference of the conduction-band wave functions between $v=1$ and $v=2$ fillings indicates that they are intrinsically different correlated states, which could be attributed to the fact that the $v=1$ state is both spin and valley polarized while the $v=2$ state can only either be spin polarized or valley polarized 
$[16,27,28,30,31]$. Theoretical studies focusing on the spatially resolved calculation of wave functions with different degeneracies could provide understanding of the broken symmetries in this system.

\section{CONCLUSIONS}

In summary, we have shown that the wave functions of the insulating state at CNP are consistent with stripe charge order [23]. The insulating states at $v=1$ and $v=2$ have distinct wave functions; their lower valence-band wave functions are delocalized while their conduction-band wave functions are localized on different sites. The coherent-driven broken symmetry states that we have observed are consistent with transport measurements [25-29] and previous STM studies [23,24,32-34] but highlight the intrinsic difference between the correlated insulating states at different commensurate fillings, which was not discovered previously. By direct measurement of the LDOS maps and the localization of these wave functions, we visualized the spatial dependence of the flat band wave functions as a function of doping level, providing a deeper understanding towards the nature of correlated states in MATBG at various commensurate fillings.

\section{ACKNOWLEDGMENTS}

The authors thank Allan H. MacDonald for valuable theoretical discussions. The work at the University of Arizona was supported by the National Science Foundation under Grants No. DMR-1708406 and No. EECS-1607911 and the Army Research Office under Grant No. W911NF-14-1-0653. K.W. and T.T. acknowledge support from the EMEXT Element Strategy Initiative to Form Core Research Center, Grant No. JPMXP0112101001 and the CREST(JPMJCR15F3), JST.

\section{APPENDIX}

\section{Tip gating correction for $d I / d V$ measurements}

In the moiré region close to the magic angle, quantum dots can be induced by the formation of insulating states [33]; they appear as constant density resonance lines in Fig. 5, indicated by the red arrows. In the uncorrected image, Fig. 5(a), the constant density lines are sloped due to tip-gating effects. In Fig. 5(b) we correct the tip gating effect by shifting the $d I / d V$ curves to make the constant density lines vertical; thus the bias voltage and carrier density are decoupled in the corrected image. The tip-gating correction does not change the appearance of the correlated effects as we discussed in the main text; however, it enables more accuate measurement of bandwidths.

\section{Non-magic-angle spectroscopy}

To show that the correlation effects we discussed in the main text are only present in the moiré flat bands close to the magic angle, Fig. 6 shows the gate-dependent STS spectroscopies for $1.30^{\circ}$ and $2.31^{\circ}$ twist angles. Consistent with previous observations [36], the Van Hove singularity (VHS) peaks are still present for both areas; however, in contrast to the magic angle area, there is no broadening of the peaks when

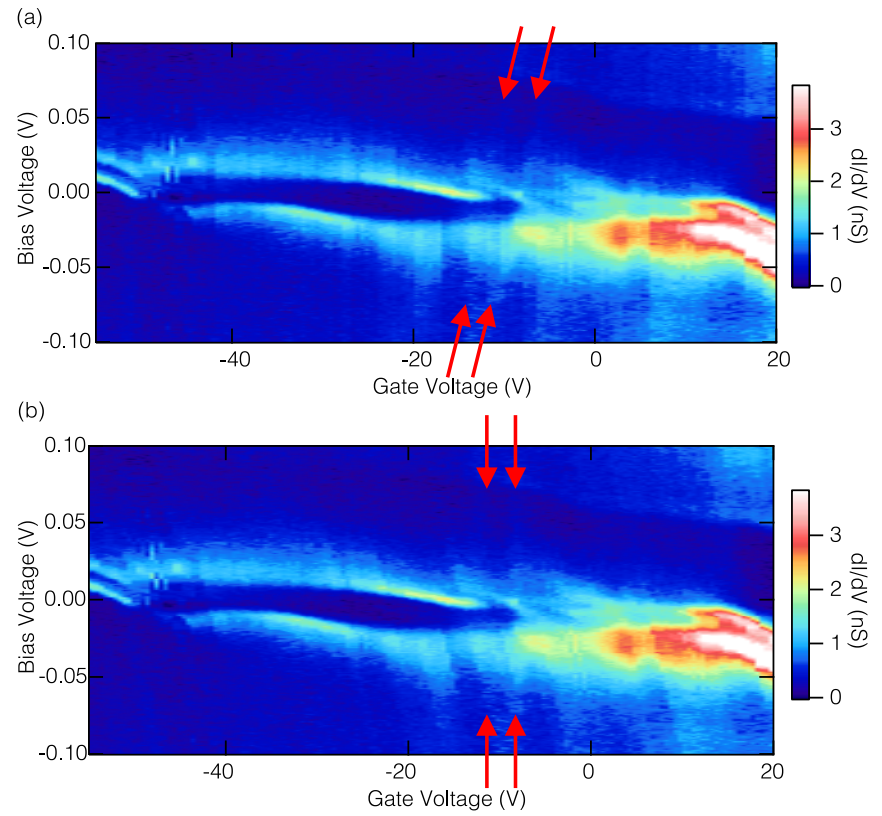

FIG. 5. (a) Gate-dependent STS spectroscopy on the $A A$ site of the $1.07^{\circ}$ moiré superlattice before the tip gating correction. (b) Gatedependent STS spectroscopy after the bias voltage is decoupled from the carrier density.

they are partially filled. The separation between the peaks is almost constant for all gate voltages, and no significant distortion of the peaks occurs near commensurate fillings.

\section{Angle dependence of bandwidths and separation between bands}

In the main text, we have shown the total bandwidth of both flat bands decreases with angle and the difference
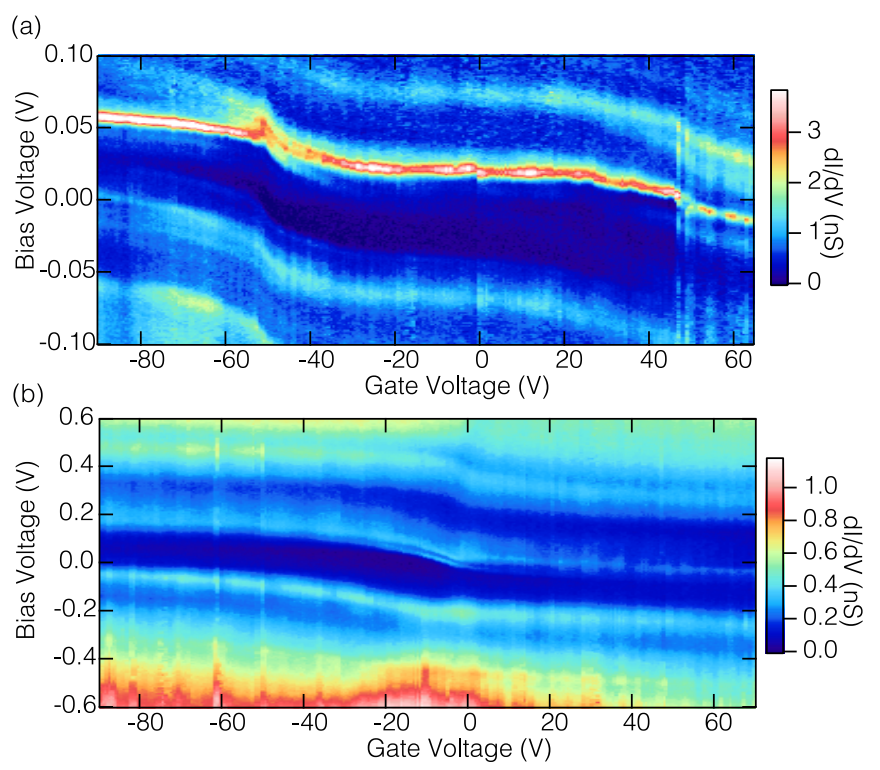

FIG. 6. (a) Gate-dependent STS spectroscopy on the $A A$ site of the $1.30^{\circ}$ twist angle device. (b) Gate-dependent STS spectroscopy on the $A A$ site of the $2.31^{\circ}$ twist angle device. 
(a)

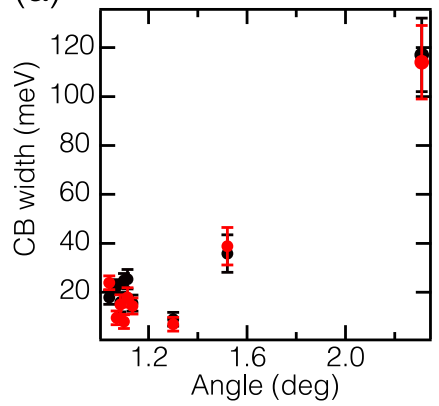

(d)

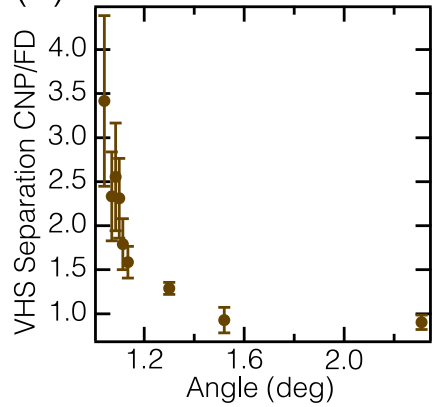

(b)

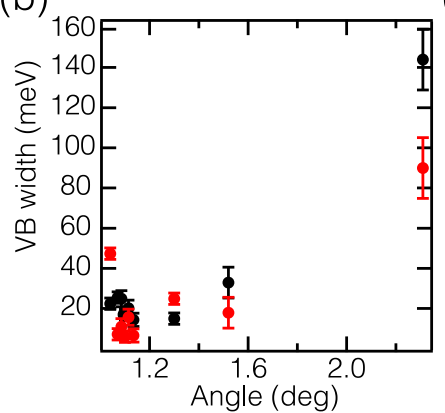

(e)

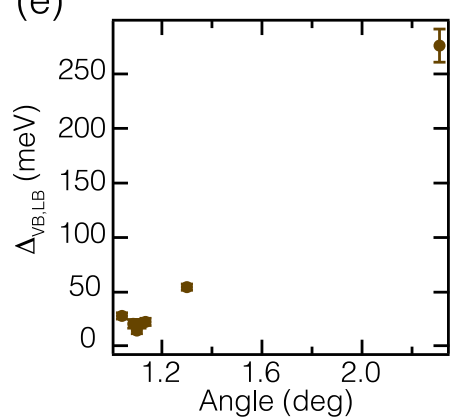

(c)

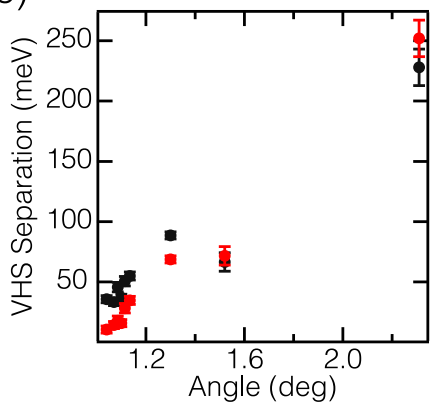

(f)

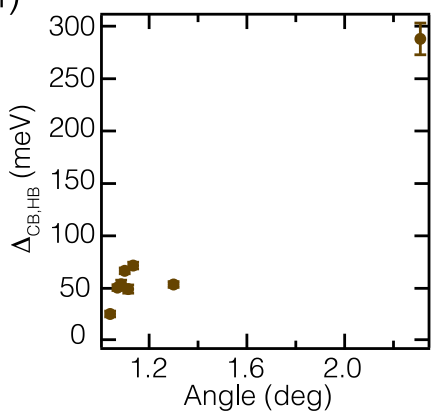

FIG. 7. Angle dependence of (a) the conduction-band width, (b) the valence-band width, and (c) the VHS separation. The red circles are when the band is fully depleted, and the black circles are at the CNP. Angle dependence of (d) the ratio of the VHS separation between CNP and FD, (e) the gap between the valence flat band and the lower dispersive band, and (f) the gap between the conduction flat band and the higher dispersive band.

between the total bandwidth at the CNP and FD is maximum around the magic angle. Figures 7(a) and 7(b) show that the bandwidths of the individual bands also decrease with angle, but the difference between the CNP and FD do not show clear indication of enhancement around the magic angle, suggesting that the enhanced separation at CNP originates from interband interactions. Figure 7(c) shows the VHS separation decreases with angle; the ratio of VHS separation at the CNP compared to FD also increases at small angle as shown in Fig. 7(d). Figures 7(e) and 7(f) show the gap between the valence flat band and lower dispersive band, and the gap between the conduction flat band and the higher dispersive band, both decrease with angle, consistent with theoretical predictions [6] and previous STS measurements [33].

\section{Tip-induced asymmetry in gate-dependent $d I / d V$ measurements}

The gate-dependent STS measurements often show asymmetric shapes in $d I / d V$ as a function of gate voltages; this tip band bending effect can be explained by the difference in the work function between the STM tip and the sample [32]. With different tip conditions, we observed symmetric spectroscopy in Fig. 8(a) and asymmetric spectroscopy bending towards the opposite direction in Fig. 8(b) as compared with Fig. 5(b).

The strength of $d I / d V$ is also asymmetric as a function of gate voltage; this is affected by the set point of the bias voltage as discussed in the main text. Figures 9(a) and 9(b) shows the gate-dependent STS spectroscopy on the same spot on the sample and with the same tip conditions but with set points of opposite sign; as a result, the $d I / d V$ is enhanced in different gate voltage ranges of the image.
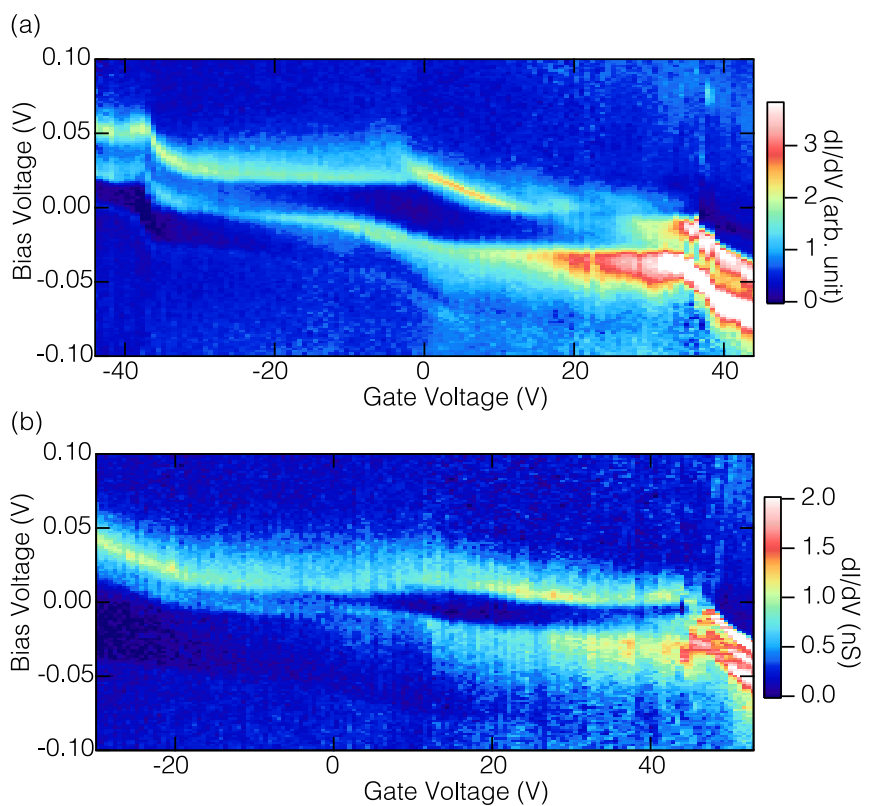

FIG. 8. (a) Gate-dependent STS spectroscopy on the $A A$ site of the $1.11^{\circ}$ moiré superlattice without strong tip band bending effect. (b) Gate-dependent STS spectroscopy on the $A A$ site of the $1.09^{\circ}$ moiré superlattice with strong tip band bending effect. 

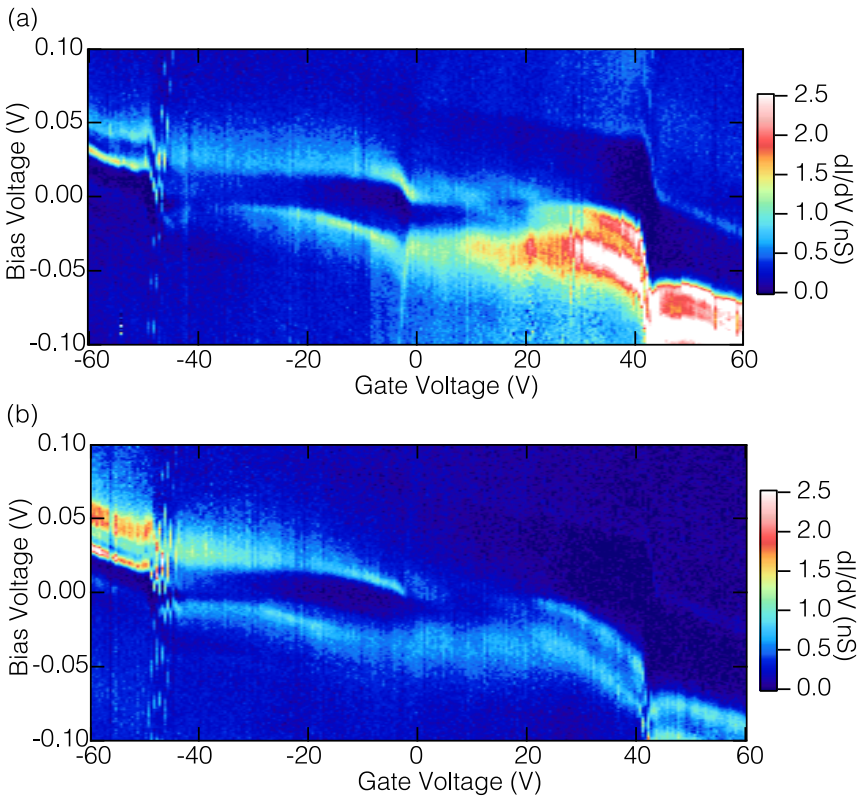

FIG. 9. (a) Gate-dependent STS spectroscopy on the $A A$ site of the $1.08^{\circ}$ moiré superlattice with a set point voltage of $0.15 \mathrm{~V}$. (b) Gate-dependent STS spectroscopy on the $A A$ site of the $1.08^{\circ}$ moiré superlattice with a set point voltage of $-0.15 \mathrm{~V}$.

\section{LDOS maps at different energy and filling levels}

In addition to Fig. 4, Fig. 10(a) shows a series of LDOS maps around the flat band energies for $v=1$. To quantify the $C_{3}$ rotational symmetry breaking, we follow the definition of energy-dependent anisotropy $A(E)$ as [24]

$$
\begin{aligned}
A(E)= & \frac{1}{3} \sum_{\text {unit cell }}\left[\frac{\left|I_{0^{\circ}}(E)-I_{120^{\circ}}(E)\right|}{I_{0^{\circ}}(E)+I_{120^{\circ}}(E)}+\frac{\left|I_{120^{\circ}}(E)-I_{240^{\circ}}(E)\right|}{I_{120^{\circ}}(E)+I_{240^{\circ}}(E)}\right. \\
& \left.+\frac{\left|I_{240^{\circ}}(E)-I_{0^{\circ}}(E)\right|}{I_{240^{\circ}}(E)+I_{0^{\circ}}(E)}\right],
\end{aligned}
$$

where $I_{0^{\circ}}(E)$ is the spatial LDOS profile when the image is aligned with the unit cell; $I_{120^{\circ}}(E)$ and $I_{240^{\circ}}(E)$ correspond to the spatial LDOS profile when $I_{0^{\circ}}(E)$ is rotated $120^{\circ}$ and $240^{\circ}$, respectively. As seen in Fig. 10(b), when the wave functions are delocalized near the filled valence flat band energies $\left(V_{b}=-36\right.$ to $\left.-32 \mathrm{mV}\right)$, their anisotropy is comparable to the anisotropy of the topography image [Fig. 2(b)], which is $2.94 \%$. In contrast, the anisotropies for the partially filled conduction flat band wave functions are much larger. To quantify how centered the wave functions are at the $A A$ site, we define the radial distribution by averaging the LDOS over a circle at each radial distance from the center of the $A A$ site, then normalize so that the summation over the full unit cell is 1 . Figure 10 (c) shows the radial distribution of the wave functions; the gray area means that the LDOS maps are not available in Fig. 10(a). Here the delocalized wave functions show uniform distribution, while the unoccupied valence flat band states $\left(V_{b}=1\right.$ to $\left.5 \mathrm{mV}\right)$ are gradually shifted away from the $A A$ sites. To quantify the overall localization of the wave functions, we plot in Fig. 10(d) a histogram at each bias voltage of the normalized LDOS for each wave function in Fig. 10(a), together with the STS spectroscopy taken at the center of the $A A$ site plotted against the right axis. When the histogram is more concentrated as a function

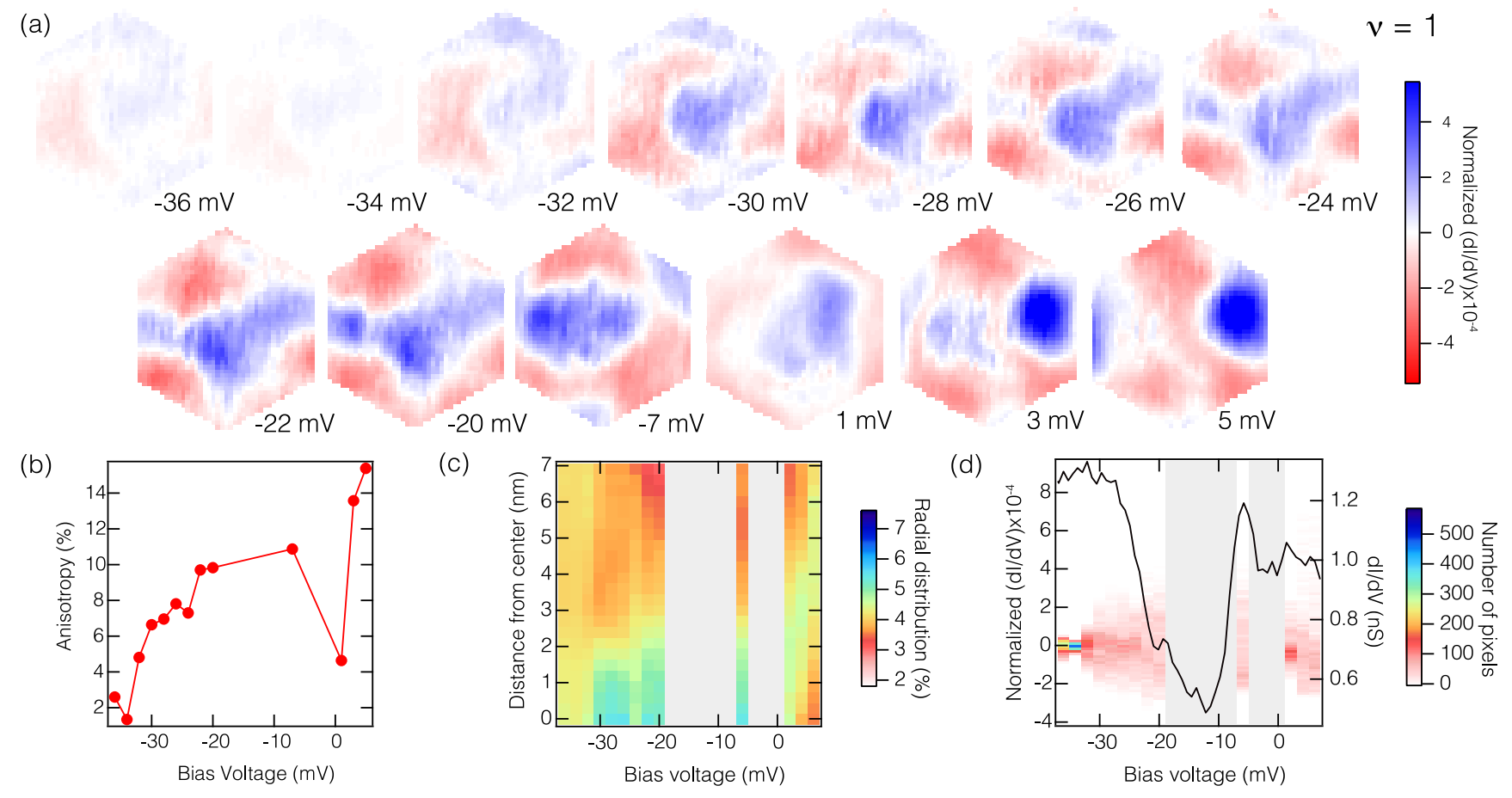

FIG. 10. (a) LDOS maps at different energies for $v=1$. (b) Anisotropy extracted from (a) as a function of bias voltage. (c) Radial distribution extracted from (a) as a function of bias voltage. (d) Color plot plotted against left axis: histogram of (a); black curve ploted against right axis: STS spectroscopy on the center of the $A A$ site. 
(a)
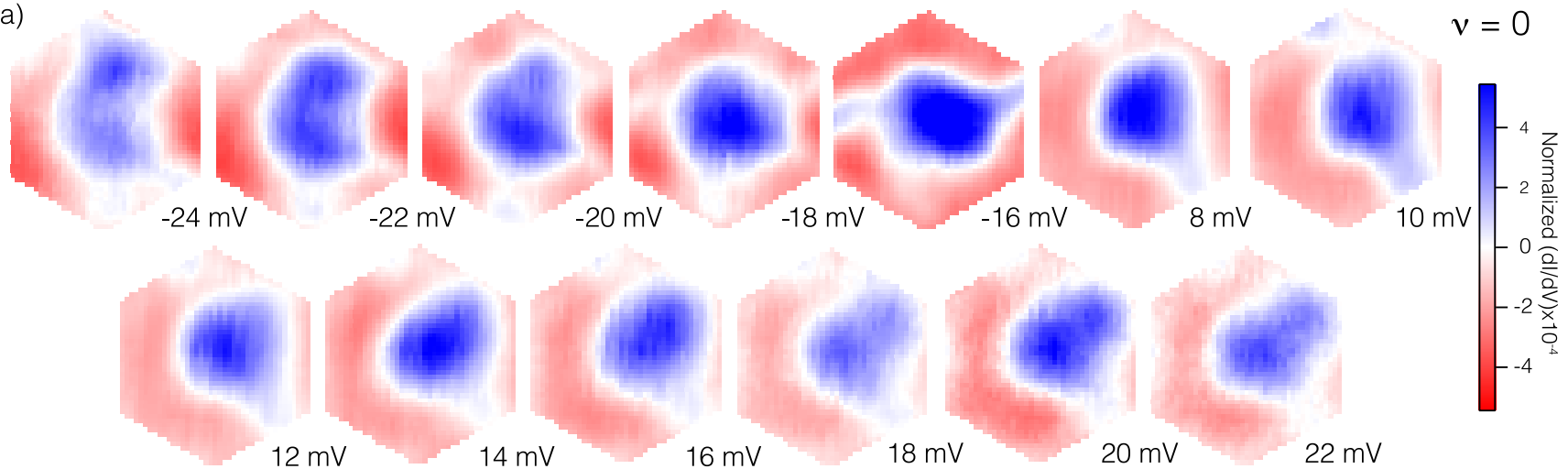

(b)

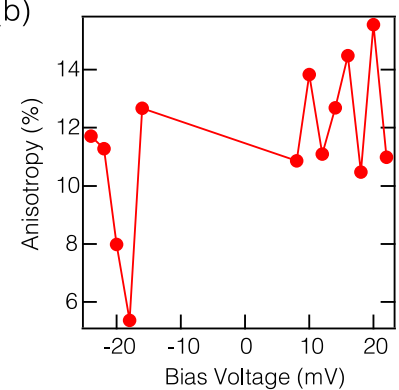

(c)

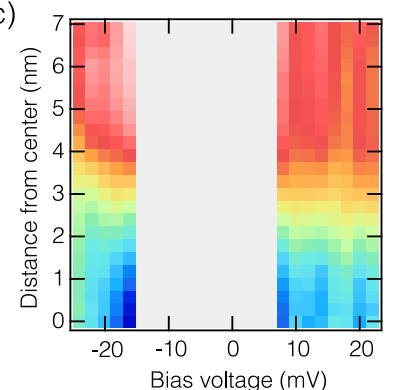

(d)

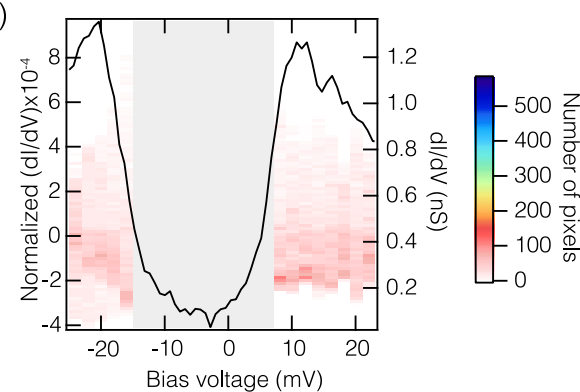

FIG. 11. (a) LDOS maps at different energies for $v=0$. (b) Anisotropy extracted from (a) as a function of bias voltage. (c) Radial distribution extracted from (a) as a function of bias voltage. (d) Color plot plotted against left axis: histogram of (a); black curve plotted against right axis: STS spectroscopy on the center of the $A A$ site.

of LDOS, the wave function is more uniform or delocalized, because most pixels in the image have the same intensity. When the histogram has a broader distribution of LDOS values, there is more contrast in the image, which means the wave function is more localized. Figure 10(d) shows that the filled valence flat band states are strongly delocalized,

(a)

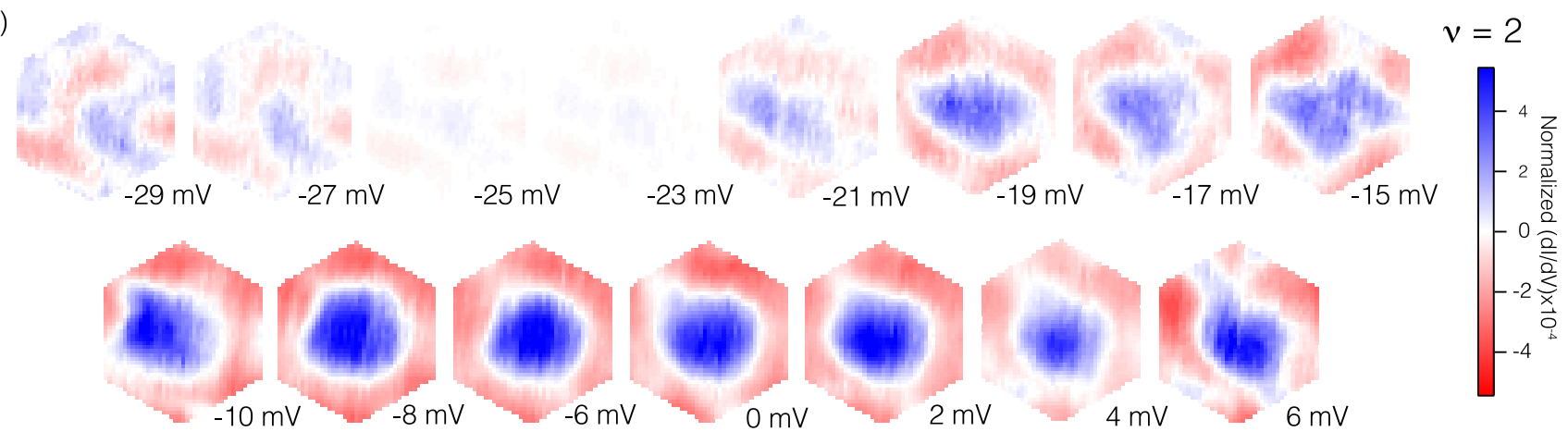

(b)

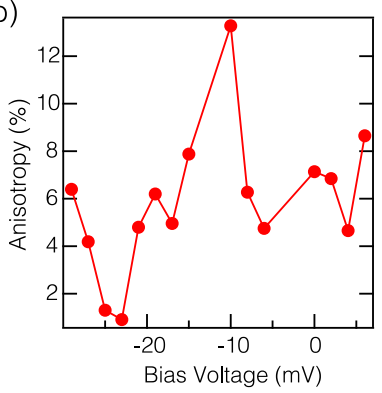

(c)

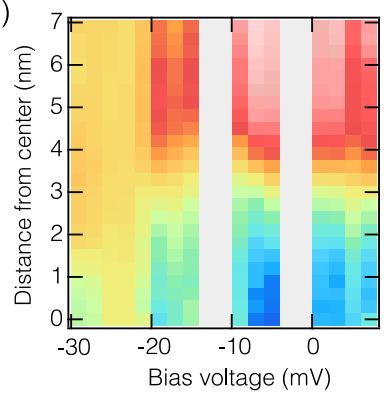

(d)

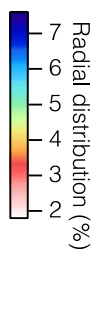

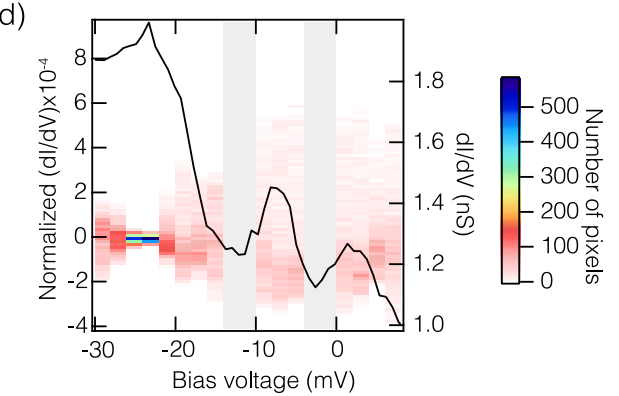

FIG. 12. (a) LDOS maps at different energies for $v=2$. (b) Anisotropy extracted from (a) as a function of bias voltage. (c) Radial distribution extracted from (a) as a function of bias voltage. (d) Color plot plotted against left axis: histogram of (a); black curve plotted against right axis: STS spectroscopy on the center of the $A A$ site. 
(a)

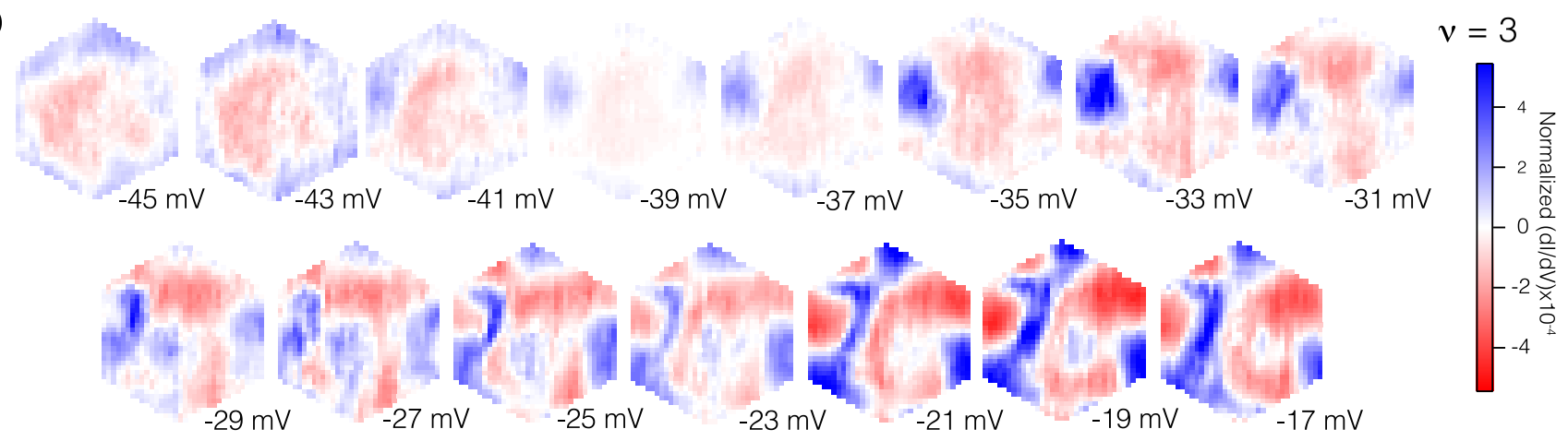

(b)

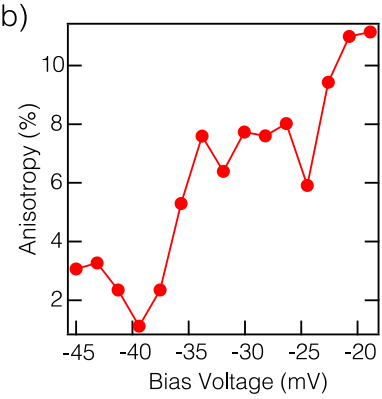

(c)

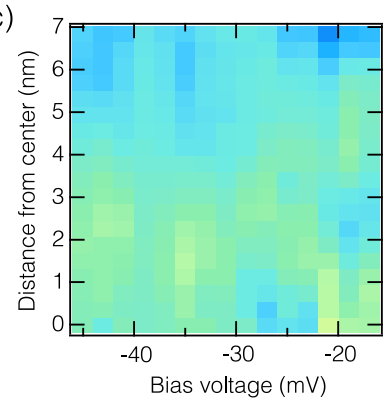

(d)

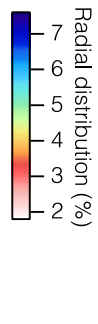

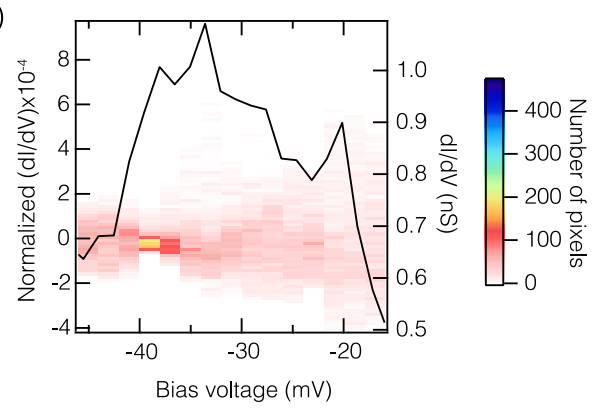

FIG. 13. (a) LDOS maps at different energies for $v=3$. (b) Anisotropy extracted from (a) as a function of bias voltage. (c) Radial distribution extracted from (a) as a function of bias voltage. (d) Color plot plotted against left axis: histogram of (a); black curve plotted against right axis: STS spectroscopy on the center of the $A A$ site.

while the partially filled conduction flat band states are still localized.

The same analysis is applied for $v=0,2,3,4$, and -4 and is shown in Figs. 11-15. For $v=0,4$, and -4 , the wave functions are always localized on the $A A$ sites. For $v=2$ and 3 , the filled valence flat band is delocalized, consistent with our findings in the main text. The partially filled conduction flat band is still localized on $A A$ for $v=2$ but localized on a

(a)
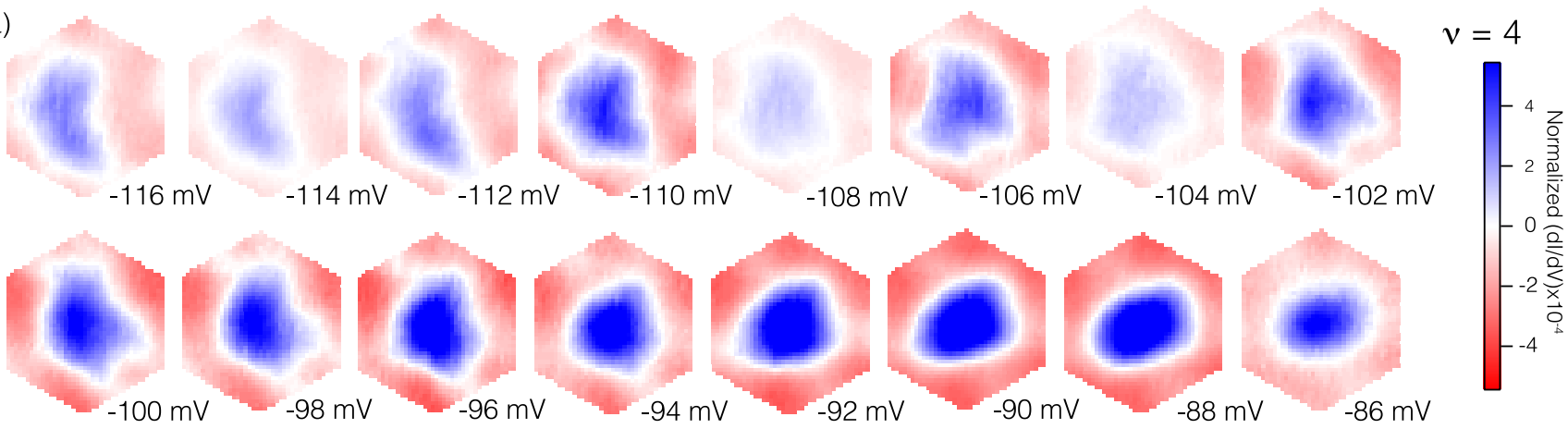

(b)

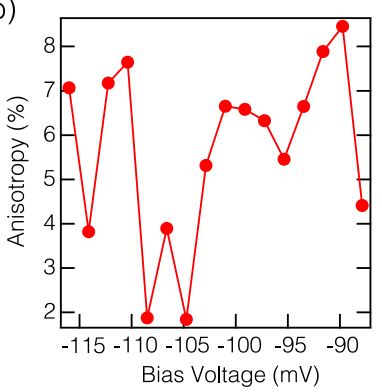

(c)

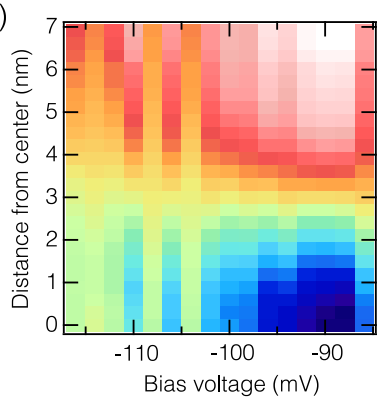

(d)

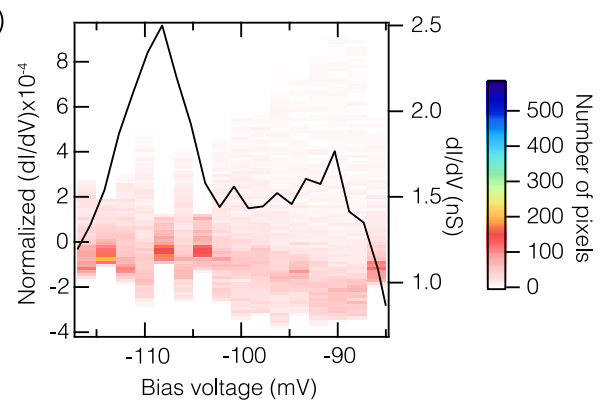

FIG. 14. (a) LDOS maps at different energies for $v=4$. (b) Anisotropy extracted from (a) as a function of bias voltage. (c) Radial distribution extracted from (a) as a function of bias voltage. (d) Color plot plotted against left axis: histogram of (a); black curve plotted against right axis: STS spectroscopy on the center of the $A A$ site. 

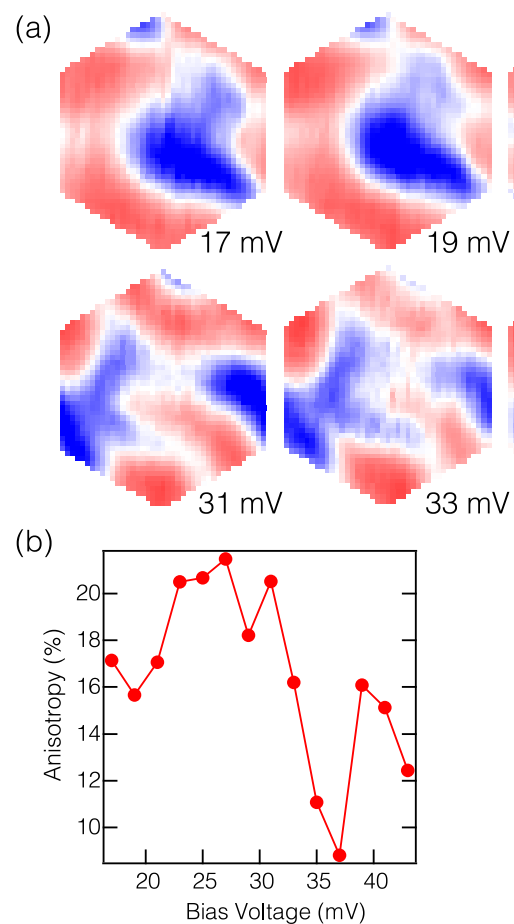

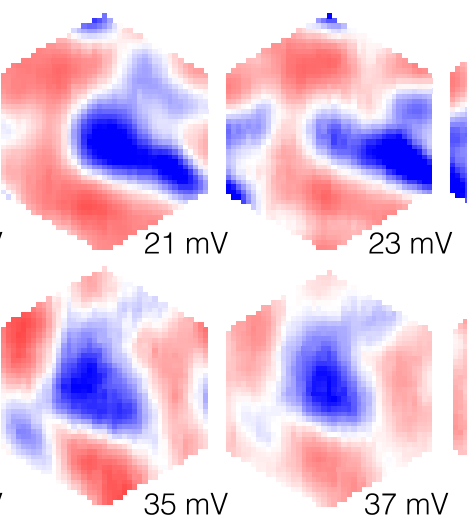

(c)

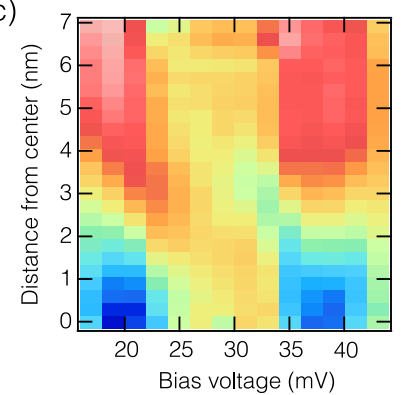

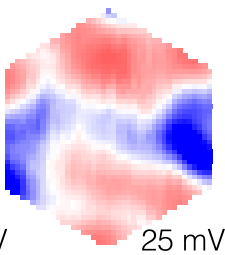
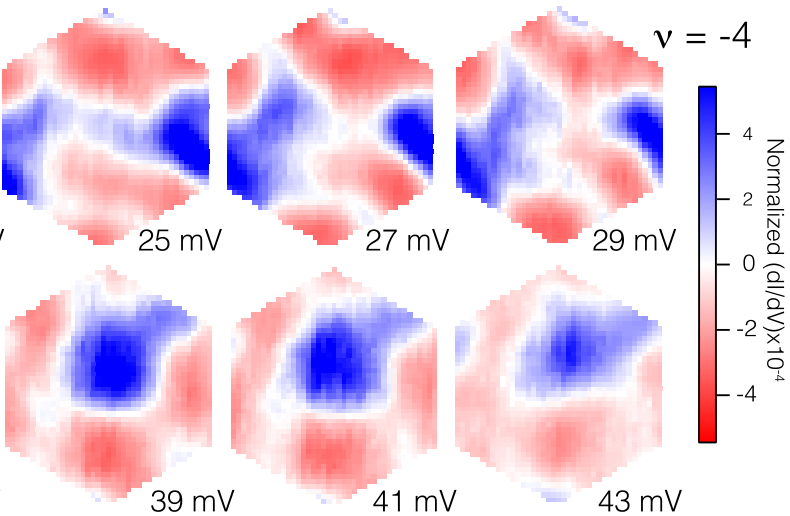

(d)
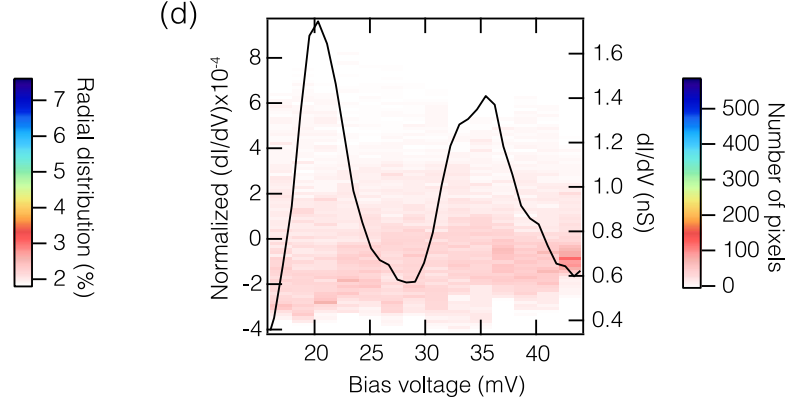

FIG. 15. (a) LDOS maps at different energies for $v=-4$. (b) Anisotropy extracted from (a) as a function of bias voltage. (c) Radial distribution extracted from (a) as a function of bias voltage. (d) Color plot plotted against left axis: histogram of (a); black curve plotted against right axis: STS spectroscopy on the center of the $A A$ site.

different location for $v=3$. Due to tip-induced band bending, all flat band energies are below the Fermi level for $v=3$.
However, their wave functions still show similar behavior as the wave functions for $v=1$.
[1] R. Bistritzer and A. H. MacDonald, Proc. Natl. Acad. Sci. USA 108, 12233 (2011).

[2] J. Jung, A. Raoux, Z. Qiao, and A. H. MacDonald, Phys. Rev. B 89, 205414 (2014).

[3] J. M. B. Lopes dos Santos, N. M. R. Peres, and A. H. Castro Neto, Phys. Rev. B 86, 155449 (2012).

[4] S. Carr, S. Fang, Z. Zhu, and E. Kaxiras, Phys. Rev. Res. 1, 013001 (2019).

[5] M. Koshino, N. F. Q. Yuan, T. Koretsune, M. Ochi, K. Kuroki, and L. Fu, Phys. Rev. X 8, 031087 (2018).

[6] N. N. T. Nam and M. Koshino, Phys. Rev. B 96, 075311 (2017).

[7] S. Carr, S. Fang, H. C. Po, A. Vishwanath, and E. Kaxiras, Phys. Rev. Res. 1, 033072 (2019).

[8] T. M. R. Wolf, J. L. Lado, G. Blatter, and O. Zilberberg, Phys. Rev. Lett. 123, 096802 (2019).

[9] F. Wu, A. H. MacDonald, and I. Martin, Phys. Rev. Lett. 121, 257001 (2018).

[10] Y. Cao, D. Chowdhury, D. Rodan-Legrain, O. Rubies-Bigorda, K. Watanabe, T. Taniguchi, T. Senthil, and P. Jarillo-Herrero, Phys. Rev. Lett. 124, 076801 (2020).

[11] H. C. Po, L. Zou, A. Vishwanath, and T. Senthil, Phys. Rev. X 8, 031089 (2018).

[12] J. Kang and O. Vafek, Phys. Rev. Lett. 122, 246401 (2019).

[13] K. Seo, V. N. Kotov, and B. Uchoa, Phys. Rev. Lett. 122, 246402 (2019).
[14] N. F. Q. Yuan and L. Fu, Phys. Rev. B 98, 045103 (2018).

[15] M. Ochi, M. Koshino, and K. Kuroki, Phys. Rev. B 98, 081102(R) (2018).

[16] J. Liu and X. Dai, arXiv:1911.03760.

[17] F. Xie, Z. Song, B. Lian, and B. A. Bernevig, Phys. Rev. Lett. 124, 167002 (2020).

[18] X. Hu, T. Hyart, D. I. Pikulin, and E. Rossi, Phys. Rev. Lett. 123, 237002 (2019).

[19] A. Julku, T. J. Peltonen, L. Liang, T. T. Heikkilä, and P. Törmä, Phys. Rev. B 101, 060505(R) (2019).

[20] M. Serlin, C. L. Tschirhart, H. Polshyn, Y. Zhang, J. Zhu, K. Watanabe, T. Taniguchi, L. Balents, and A. F. Young, Science 367, 900 (2020).

[21] H. Polshyn, M. Yankowitz, S. Chen, Y. Zhang, K. Watanabe, T. Taniguchi, C. R. Dean, and A. F. Young, Nat. Phys. 15, 1011 (2019).

[22] S. Liu, E. Khalaf, J. Y. Lee, and A. Vishwanath, arXiv:1905.07409.

[23] Y. Jiang, X. Lai, K. Watanabe, T. Taniguchi, K. Haule, J. Mao, and E. Y. Andrei, Nature 573, 91 (2019).

[24] A. Kerelsky, L. J. McGilly, D. M. Kennes, L. Xian, M. Yankowitz, S. Chen, K. Watanabe, T. Taniguchi, J. Hone, C. Dean, A. Rubio, and A. N. Pasupathy, Nature 572, 95 (2019).

[25] Y. Cao, V. Fatemi, A. Demir, S. Fang, S. L. Tomarken, J. Y. Luo, J. D. Sanchez-Yamagishi, K. Watanabe, T. Taniguchi, E. 
Kaxiras, R. C. Ashoori, and P. Jarillo-Herrero, Nature 556, 80 (2018).

[26] Y. Cao, V. Fatemi, S. Fang, K. Watanabe, T. Taniguchi, E. Kaxiras, and P. Jarillo-Herrero, Nature 556, 43 (2018).

[27] M. Yankowitz, S. Chen, H. Polshyn, Y. Zhang, K. Watanabe, T. Taniguchi, D. Graf, A. F. Young, and C. R. Dean, Science 363, 1059 (2019).

[28] X. Lu, P. Stepanov, W. Yang, M. Xie, M. A. Aamir, I. Das, C. Urgell, K. Watanabe, T. Taniguchi, G. Zhang, A. Bachtold, A. H. MacDonald, and D. K. Efetov, Nature 574, 653 (2019).

[29] A. L. Sharpe, E. J. Fox, A. W. Barnard, J. Finney, K. Watanabe, T. Taniguchi, M. A. Kastner, and D. GoldhaberGordon, Science 365, 605 (2019).

[30] M. Xie and A. H. MacDonald, Phys. Rev. Lett. 124, 097601 (2020).

[31] N. Bultinck, E. Khalaf, S. Liu, S. Chatterjee, A. Vishwanath, and M. P. Zaletel, arXiv:1911.02045 [Phys. Rev. X (to be published)].
[32] Y. Xie, B. Lian, B. Jäck, X. Liu, C.-L. Chiu, K. Watanabe, T. Taniguchi, B. A. Bernevig, and A. Yazdani, Nature 572, 101 (2019).

[33] Y. Choi, J. Kemmer, Y. Peng, A. Thomson, H. Arora, R. Polski, Y. Zhang, H. Ren, J. Alicea, G. Refael, F. von Oppen, K. Watanabe, T. Taniguchi, and S. Nadj-Perge, Nat. Phys. 15, 1174 (2019).

[34] D. Wong, K. P. Nuckolls, M. Oh, B. Lian, Y. Xie, S. Jeon, K. Watanabe, T. Taniguchi, B. A. Bernevig, and A. Yazdani, Nature 582, 198 (2020).

[35] K. Kim, M. Yankowitz, B. Fallahazad, S. Kang, H. C. P. Movva, S. Huang, S. Larentis, C. M. Corbet, T. Taniguchi, K. Watanabe, S. K. Banerjee, B. J. LeRoy, and E. Tutuc, Nano Lett. 16, 1989 (2016).

[36] D. Wong, Y. Wang, J. Jung, S. Pezzini, A. M. DaSilva, H.-Z Tsai, H. S. Jung, R. Khajeh, Y. Kim, J. Lee, S. Kahn, S. Tollabimazraehno, H. Rasool, K. Watanabe, T. Taniguchi, A. Zettl, S. Adam, A. H. MacDonald, and M. F. Crommie, Phys. Rev. B 92, 155409 (2015). 